\title{
Performance evaluation of SiPM photodetectors for PET imaging in the presence of magnetic fields
}

\author{
S. España ${ }^{\mathrm{a}, *, 1}$, L.M. Fraile ${ }^{\mathrm{a}}$, J.L. Herraiz ${ }^{\mathrm{a}}$, J.M. Udías ${ }^{\mathrm{a}}$, M. Desco ${ }^{\mathrm{b}}$, J.J. Vaquero ${ }^{\mathrm{b}}$

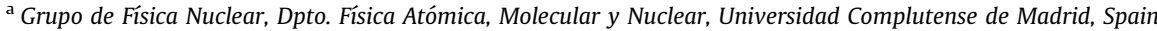 \\ ${ }^{\mathrm{b}}$ Unidad de Medicina y Cirugía Experimental, Hospital General Universitario Gregorio Marañón, Madrid, Spain
}

\section{A R T I C L E I N F O}

\section{Article history:}

Received 12 June 2009

Received in revised form

22 November 2009

Accepted 24 November 2009

Available online 2 December 2009

\section{Keywords:}

Silicon photomultiplier

Positron emission tomography

Magnetic resonance imaging

\begin{abstract}
A B S T R A C T
The multi pixel photon counter (MPPC) or silicon photomultiplier (SiPM), recently introduced as a solid state photodetector, consists of an array of Geiger mode photodiodes (microcells). It is a promising device for PET due to its potential for high photon detection efficiency (PDE) and its foreseeable immunity to magnetic fields. It is also easy to use with simple read outs, has a high gain and a small size. In this work we evaluate the in field performance of three $1 \times 1 \mathrm{~mm}^{2}$ (with 100,400 and 1600 microcells, respectively) and one $6 \times 6 \mathrm{~mm}^{2}$ (arranged as a $2 \times 2$ array) Hamamatsu MPPCs for their use in PET imaging. We examine the dependence of the energy resolution and the gain of these devices on the temperature and reverse bias voltage, when coupled to LYSO scintillator crystals under conditions that one would find in a PET system. We find that the 400 and 1600 microcells models and the $2 \times 2$ array are suitable for small size crystals, like those employed in high resolution small animal scanners. We have confirmed the good performance of these devices up to magnetic fields of $7 \mathrm{~T}$ as well as their suitability for performing PET acquisitions in the presence of fast switching gradients and high duty radiofrequency MRI sequences.
\end{abstract}

\section{Introduction}

Positron emission tomography (PET) scanners have been extensively used to investigate biochemical and pathological phenomena, to diagnose disease, and to determine prognosis after treatment. Magnetic resonance imaging (MRI) provides unsur passed soft tissue contrast and does not make use of ionizing radiation. Therefore, it is expected that combined PET/MR scanners may represent an interesting combination of modalities for biomedical imaging [1], and that these scanners will either supplement or compete with PET/CT ones for basic research and clinical applications. However, one major obstacle for combining PET and MR systems based on photomultiplier tubes (PMT) is that these are extremely sensitive to magnetic fields [2]. Indeed, PET/ MR scanners based on PMTs require the use of long optical fibers to transfer the light from the scintillation crystals located inside the MR scanner to the PMTs [3]. Due to light loses, a degradation of both energy resolution and time response occurs.

The use of semiconductor photodetectors, such as avalanche photodiodes (APD) offers an alternative to PMTs, which is

\footnotetext{
* Corresponding author. Tel.: +34913944 484; fax: +34913945193.

E-mail address: samuel@nuclear.fis.ucm.es (S. España).

${ }^{1}$ Current address: Department of Radiation Oncology, Massachusetts General Hospital and Harvard Medical School, Boston, MA, USA.
}

currently being pursued by several research groups [4]. APDs are compact and insensitive to magnetic fields, compared with PMTs, but currently available APDs have low gains, of the order of few a hundreds, and thus require sophisticated preamplifiers and/ or cooling.

Silicon photomultiplier detectors (SiPMs) offer new alterna tives for the design and construction of PET/MR scanners. These devices are solid state photodetectors consisting of an array of photodiodes (microcells) operated in Geiger mode [5]. They exhibit good photon detection efficiency (PDE) and they should not be affected by magnetic fields. They are easy to use, require simple electronics, do not need high voltage power supplies and provide high gain, all in a compact size. Their insensitivity to magnetic field makes them suitable for the development of hybrid scanners for simultaneous PET/MR studies. Several groups are studying the performance of SiPMs for their use in PET scanners, with promising results [6 8]. In this work we study the performance of several of these devices manufactured by Hamamatsu under conditions that would be found in both PET and simultaneous PET/MR scanners.

\section{Materials and methods}

In this work we evaluate three $1 \times 1 \mathrm{~mm}^{2}$ SiPM models with 100,400 and 1600 microcells and a $6 \times 6 \mathrm{~mm}^{2}$ SiPM, arranged as 
Table 1

Characteristics of the SiPMs employed in the present study [11].

\begin{tabular}{lllrr}
\hline Series & Active area $\left(\mathrm{mm}^{2}\right)$ & Number of pixels & Microcell (pixel) size $(\mu \mathrm{m})$ & P.D.E. $(\%)$ \\
\hline S10362-11-100P & $1 \times 1$ & 100 & 100 & 78.5 \\
S10362-11-050P & $1 \times 1$ & 400 & 50 & 65 \\
S10362-11-025P & $1 \times 1$ & 1600 & 25 & 50 \\
MPPC-33-2 $\times 2-505900$ & $6 \times 6(2 \times 2$ array) & $3600 /$ element & 50 & 25 \\
\hline
\end{tabular}

P.D.E. values include the contributions from crosstalk and afterpulses [10].
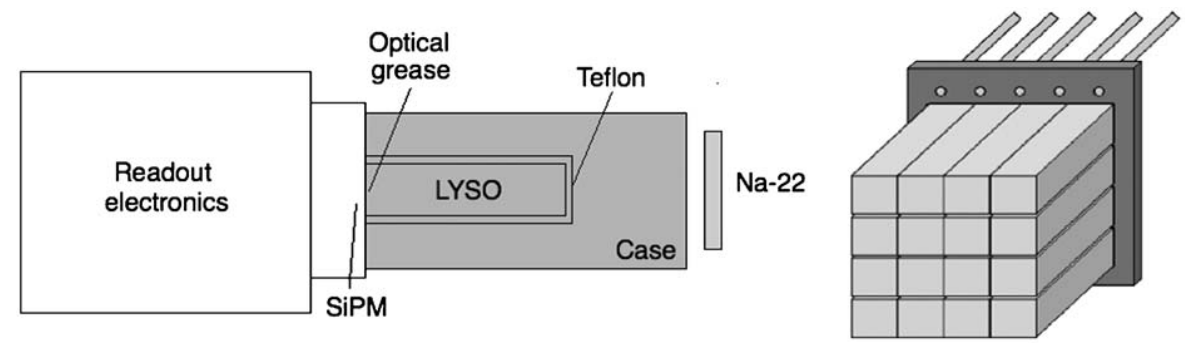

Fig. 1. Experimental setup used for $1 \times 1 \mathrm{~mm}^{2}$ SiPMs (left). Schematic experimental setup used for the SiPM array (right).

an array of $2 \times 2$ elements, with 3600 microcells per element, all of them from Hamamatsu (Table 1), for their use in PET and PET/ MR. They were coupled to LYSO crystals, some of individual size $1.5 \times 1.5 \times 12 \mathrm{~mm}^{3}$ that were coupled to the small detector, or forming an array, coupled to the larger detector, which was also tested coupled to single block crystal of $10 \times 10 \times 20 \mathrm{~mm}^{3}$. We have studied the performance of these devices under static magnetic fields of up to $7 \mathrm{~T}$ at a superconducting magnet (Bruker Corporation, Germany). The SiPM array has also been tested under simultaneous MR imaging acquisitions which made use of heavy duty magnetic field gradients and radiofrequency emissions [9].

Data acquisition was performed using a four channel Agilent 6000 series digital oscilloscope $(1 \mathrm{GHz}$ bandwidth, $2 \mathrm{GS} / \mathrm{s})$. The oscilloscope was placed in the MRI technical room, connected to the detector by $5 \mathrm{~m}$ long shielded cables. The power supply for the SiPM and the amplifier circuit was placed in the MR scanning room beyond the $5 \mathrm{G}$ line and were connected by $2 \mathrm{~m}$ long shielded cables to the detectors. All the measurements presented in this work were performed in singles mode and thus some contribution from intrinsic decay (lutetium in the scintillator) is expected. We estimated that this background contributes less than $10 \%$ to any of the measurements presented in this work.

\subsection{Single SiPMs}

Fig. 1 (left) shows the experimental setup employed to test the $1 \times 1 \mathrm{~mm}^{2}$ SiPMs. Each SiPM was coupled with optical grease to a LYSO crystal and wrapped with Teflon. A black Delrin case was used to prevent external light from reaching the SiPM.

A preamplifier was designed and built to read the SiPMs; the output pulses have a rise time of $10 \mathrm{~ns}$ and a decay time (90 10\%) of $100 \mathrm{~ns}$ (Fig. 2), which means that the shaping of the preamplifier is such that the output pulse shape is essentially dominated by the timing properties of the scintillator. This preamplifier can handle high count rates.

\subsection{SiPM array}

Readout electronics specifically tailored to this application were built in order to acquire the signals from the SiPM channels (Fig. 3). The circuit provides signals from the four channels as well

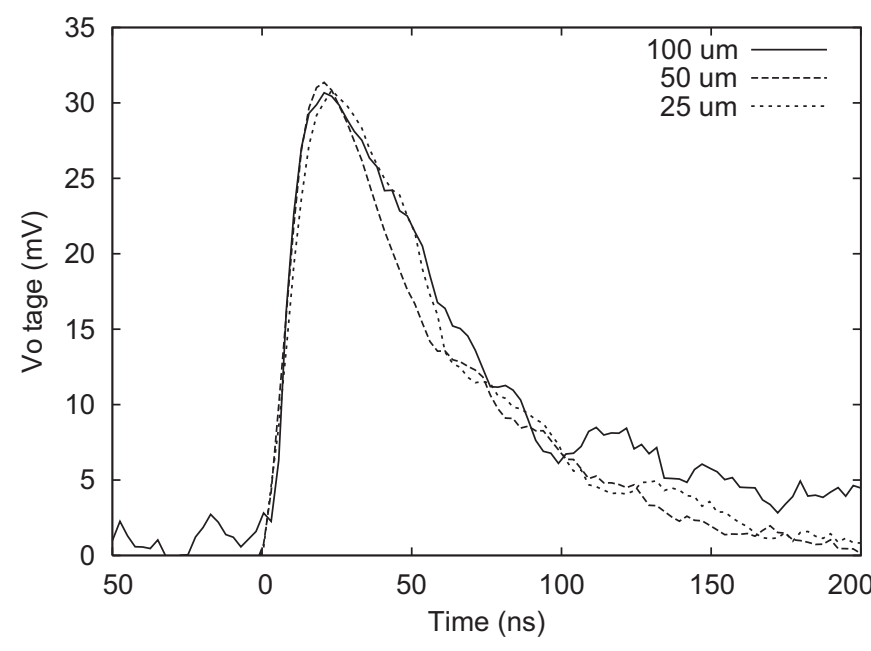

Fig. 2. Pulses obtained from the first shaping stage of the readout electronics designed for the $1 \times 1 \mathrm{~mm}^{2}$ SiPMs, for different microcell sizes. The pulses have been rescaled to approximately the same peak value.

as an additional timing signal generated from the sum of all channels, used for trigger.

Energy resolution and dependence of gain on reverse bias voltage and temperature were measured at 0 and $7 \mathrm{~T}$ using gamma photons from a ${ }^{22} \mathrm{Na}$ radioactive source and a single block LYSO crystal with dimensions $1 \times 1 \times 2 \mathrm{~cm}^{3}$, in order to acquire the energy spectrum obtained from the sum of the four channels of the SiPM array.

Further energy resolution and flood field histogram [12,13] were also measured at both 0 and $7 \mathrm{~T}$ using a $4 \times 4$ LYSO crystal matrix coupled to the $2 \times 2 \mathrm{SiPM}$ array. Each crystal element $\left(1.5 \times 1.5 \times 12 \mathrm{~mm}^{3}\right)$ was individually wrapped with Teflon (Fig. 1 , right). An ${ }^{18} \mathrm{~F}$ radioactive source was used for the measurements with the crystal array. As mentioned before, all these measure ments were done in singles mode.

The SiPM array, coupled to the crystal matrix, was also tested in presence of switching magnetic field gradients and radio frequency (RF) pulses. For this purpose we have used the RARE (TR: $2727 \mathrm{~ms}$, TE: $10.5 \mathrm{~ms}$, rare factor: 128) and MSME (TR: $1000 \mathrm{~ms}$, TE: $14 \mathrm{~ms}$ ) sequences which exhibit a very high duty 


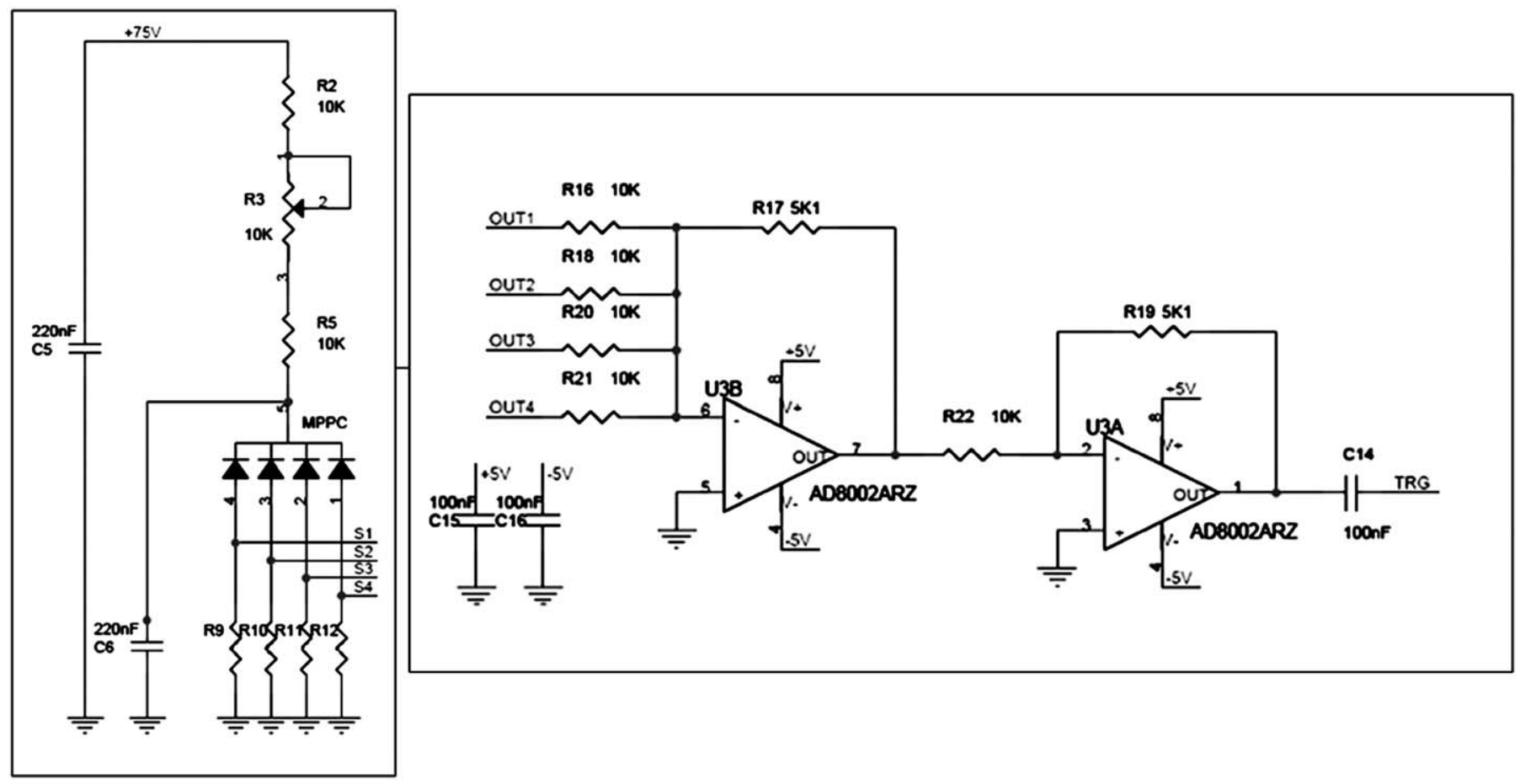

Fig. 3. Schematics of the readout electronics used for the SiPM array, based on the Analog Devices AD8002 (600 MHz bandwidth and $1200 \mathrm{~V} / \mu \mathrm{s}$ slew rate) current feedback amplifier [11].

cycle and strong magnetic field gradients, intended to test PET detectors and electronics under worst case MRI scenarios (RF peak power $=3 \mathrm{~kW}$, maximum gradient slew rate $=4500 \mathrm{~T} / \mathrm{m} / \mathrm{s}$ ).

Both the SiPM, coupled to the crystal matrix, and the electronic circuit were placed at the center of the field of view of the magnetic resonance scanner, where the amplitude of the $R F$ is maximum. A copper cylinder was built in order to shield the readout electronics from the RF. We report on the effectiveness of shielding in a specific section below. However, in order to evaluate the performance of both SiPMs and electronics in extreme conditions, unless otherwise specified, we present only the results for the unshielded case.

\section{Results for single SiPMs}

\subsection{Energy resolution}

Fig. 4 shows the energy spectra obtained from each of the three $1 \times 1 \mathrm{~mm}^{2}$ SiPMs coupled to the same LYSO crystal of $1.5 \times 1.5 \times 12 \mathrm{~mm}^{3}$, using a ${ }^{22} \mathrm{Na}$ radioactive source. These spectra have been linearized [10] to correct for the well known fact that the dynamic range of the SiPM is limited as its response saturates when most of their microcells are triggered. This behavior can be described [10] using Eq. (1), where $N_{\text {fired }}$ is the number of triggered microcells, $N_{\text {total }}$ is the number of microcells and $N_{\text {photon }}$ is the number of incident photons:

$N_{\text {fired }}=N_{\text {total }}\left[1 \exp \left(\frac{N_{\text {photon }} \times P D E}{N_{\text {total }}}\right)\right]$

We can see in Fig. 4 how the 511 keV peak is clearly resolved in all cases. However, the $1275 \mathrm{keV}$ peak is not so well resolved due to saturation for the case of the detectors with lower number of microcells and higher PDE. For the same number of emitted photons, as we are considering here, a higher number of microcells and lower PDE, yields better linearity and therefore

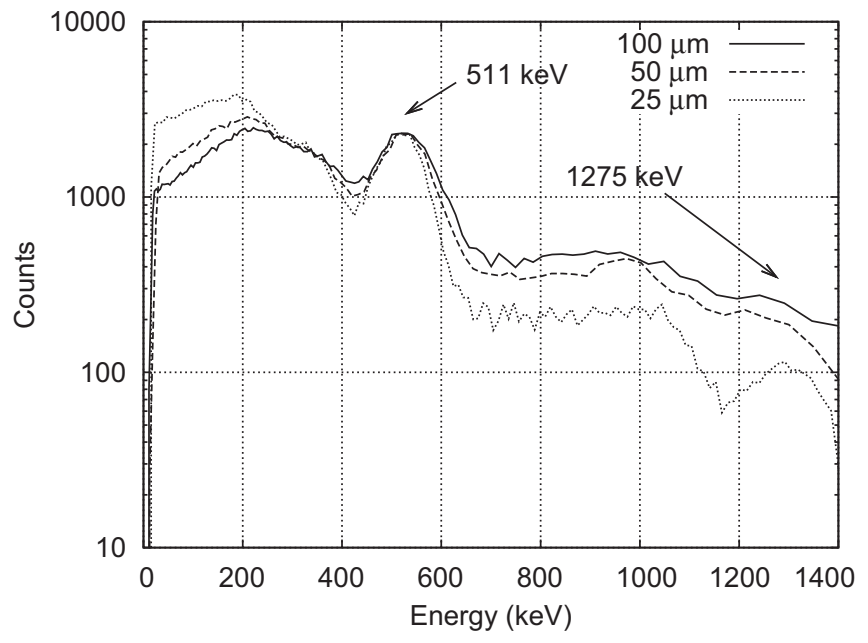

Fig. 4. Linearized (according to Eq. (1)) energy spectra obtained for the three single SiPMs from a ${ }^{22} \mathrm{Na}$ radioactive source.

the $1275 \mathrm{keV}$ peak is better observed for the SiPM with 1600 microcells (cell size: $25 \mu \mathrm{m}$ ).

Fig. 5 shows the energy resolution at $511 \mathrm{keV}$, taken as the FWHM of the peak energy (in percentage), as a function of the applied over voltage, for a constant temperature of $20^{\circ} \mathrm{C}$. The over voltage is defined as the difference (in volts) with respect to the minimum working voltage required for the detectors to work, that is, to detect pulses from the scintillator. Further, there is a maximum bias voltage where most pixels from the detectors are above breakdown voltage and then the detector shows just too many high amplitude pulses even in the absence of light, rendering the detectors not useable. These minimum and maximum working voltages are quoted in Table 2 . This table helps to determine the range of useable bias voltage under realistic PET imaging conditions. To estimate the FWHM, the 


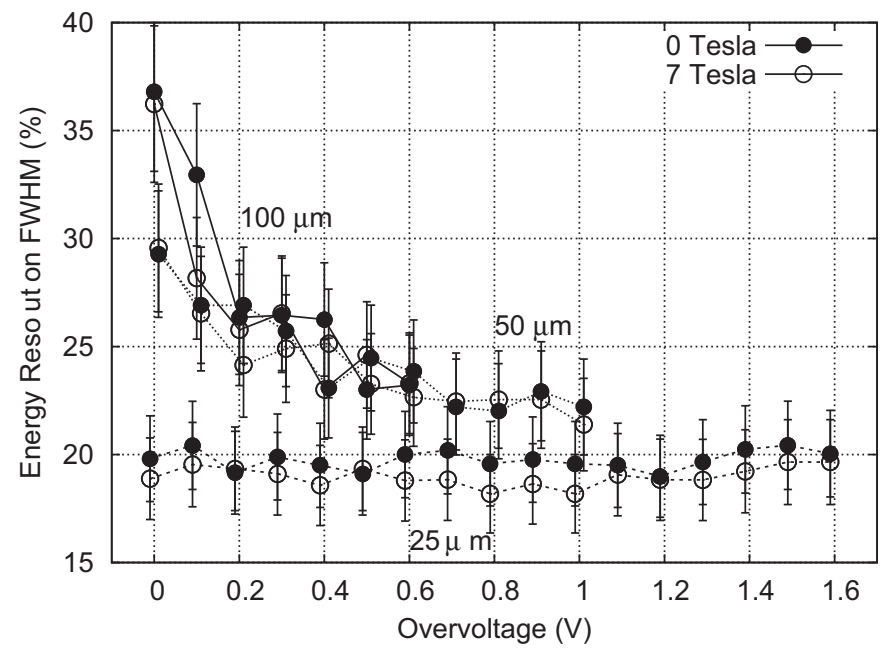

Fig. 5. Energy resolution as a function of overvoltage for the three SiPMs. Solid circles correspond to the results without magnetic field, while empty circles show the results with magnetic field. Solid line joins the data for the 100 microcells detector, dotted line for the 400 microcell detector and dashed lines for the 1600 microcell detector.

Table 2

Minimum and maximum working voltage (at $20^{\circ} \mathrm{C}$ ) indicating the range of useful bias voltage for the $1 \times 1 \mathrm{~mm}^{2}$ SiPMs and the SiPM array.

\begin{tabular}{lll}
\hline & $\begin{array}{l}\text { Minimum working } \\
\text { voltage }(\mathrm{V})\end{array}$ & $\begin{array}{l}\text { Maximum working } \\
\text { voltage }(\mathrm{V})\end{array}$ \\
\hline 100 microcells & 68.1 & 68.7 \\
400 microcells & 68.6 & 69.6 \\
1600 microcells & 70.3 & 71.9 \\
$2 \times 2$ array & 68.5 & 71.5 \\
\hline
\end{tabular}

$511 \mathrm{keV}$ peak was located and fit to a Gaussian shape. The confidence interval (65\%) for the parameters of the Gaussian obtained from the fit yields estimates of the error in the FWHM, represented by error bars in Fig. 5 .

The best resolution was obtained for the SiPMs with 1600 and 400 microcells ( 25 and $50 \mu \mathrm{m}$ ). It is also worth noticing that the FWHM for the SiPM with 1600 microcells $(25 \mu \mathrm{m})$ did not significantly change with bias voltage, while also exhibiting the larger useable bias voltage range. Both measurements were performed at $20^{\circ} \mathrm{C}$, with and without magnetic field. As in previous figure, no significant effect of magnetic field was found.

\subsection{Reverse voltage}

In Fig. 6, relative gains for the same series of measurements shown in Fig. 5 are also displayed. We define relative gains by comparing the position (channel) of the $511 \mathrm{keV}$ peak, after linearizing the spectra, for several bias voltages, taking as a reference (or $100 \%$ value), the one corresponding to the maximum practical achievable gain for each detector. The main purpose of this measurement is not to measure absolute gains of the devices that have been reported elsewhere [10], but rather to determine whether there is any dependence of this gain with the applied magnetic field. The slope of the gain against bias voltage is smallest for the SiPM with 1600 microcells $(25 \mu \mathrm{m})$, followed by those with $400(50 \mu \mathrm{m})$ and finally by the one of 100 microcells $(100 \mu \mathrm{m})$. This is probably due to a combination of several effects. In one side the microcell capacitance is larger for a larger cell size, thus producing stronger dependence of the gain for each cell on

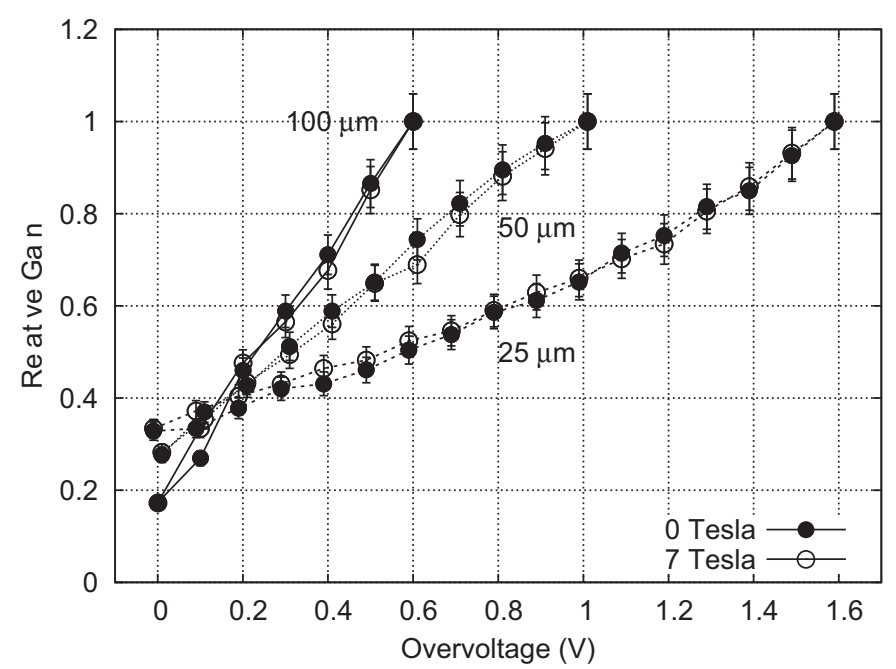

Fig. 6. Relative gain as a function of overvoltage for the $1 \times 1 \mathrm{~mm}^{2}$ SiPMs. Symbols and lines as in Fig. 5

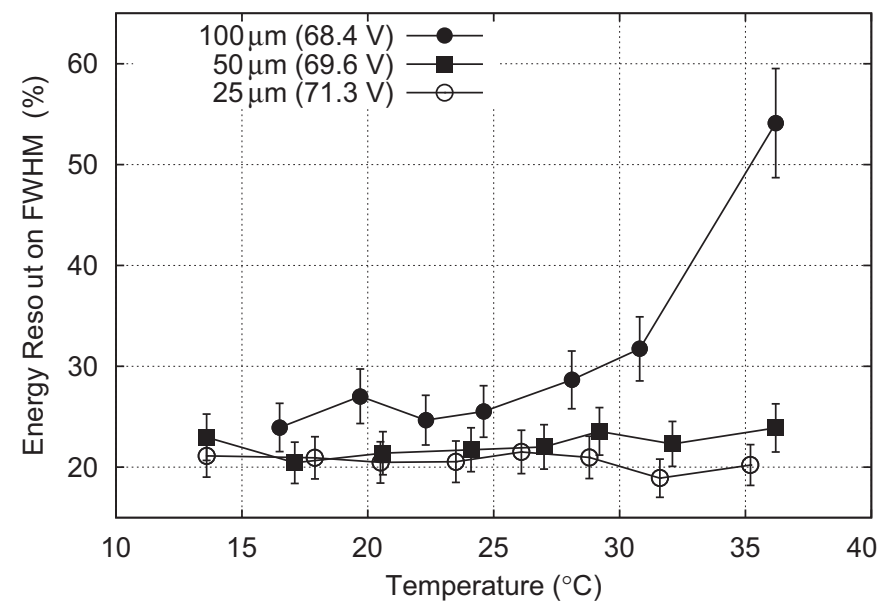

Fig. 7. Energy resolution as a function of temperature.

the voltage [14], on the other hand, because for larger number of cells there is also better linearity, that is, for the detector with larger number of microcells. If there is nonlinearity in the detector, it would exponentially enhance (Eq. (1)) the dependence of the gain with the bias voltage.

Energy resolution at $511 \mathrm{keV}$ was also measured at a fixed voltage for each SiPM, as a function of temperature. This is presented in Fig. 7. SiPMs with 1600 and 400 microcells (25 and $50 \mu \mathrm{m})$ again exhibited the most stable behavior.

\subsection{Temperature}

The variation of relative gain with temperature also shows uniform behavior for the 400 and 1600 microcells SiPMs (Fig. 8). We can observe that the gain drops with raising temperatures, which is consistent with the expected increase of the breakdown voltage as temperature becomes higher [15]. This increase in the breakdown voltage with temperature would decrease the number of cells contributing to the signal for the same amount of light and thus would cause both a smaller signal as well as poorer resolution. Further, single cell gain varies linearly with overvoltage, according to the following formula [16]:

$G=\left(V_{\text {bias }} \quad V_{\text {breakdown }}\right) \times C / q$ 


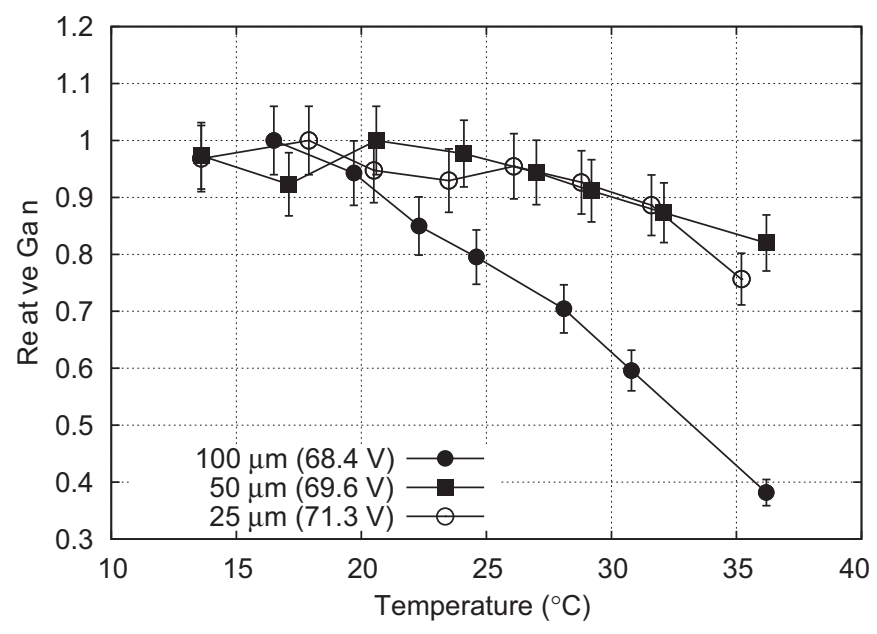

Fig. 8. Relative gain as a function of temperature.

Table 3

Summary of gain variation results for single SiPM.

\begin{tabular}{lllll}
\hline & \multicolumn{3}{l}{ Constant $T$} & \multicolumn{2}{l}{ Constant $V$} \\
\cline { 2 - 3 } & $0 \mathrm{~T}$ & $7 \mathrm{~T}$ & $0 \mathrm{~T}$ \\
\hline 100 microcells $(100 \mu \mathrm{m})$ & $230 \% \mathrm{~V}^{1}$ & $235 \% \mathrm{~V}^{1}$ & $-2.7 \%^{\circ} \mathrm{C}^{1}$ \\
400 microcells $(50 \mu \mathrm{m})$ & $110 \% \mathrm{~V}^{1}$ & $110 \% \mathrm{~V}^{1}$ & $-0.7 \%^{\circ} \mathrm{C}^{1}$ \\
1600 microcells $(25 \mu \mathrm{m})$ & $70 \% \mathrm{~V}^{1}$ & $75 \% \mathrm{~V}^{1}$ & $-1.0 \%{ }^{\circ} \mathrm{C}^{1}$ \\
\hline
\end{tabular}

where $G$ is the gain of individual cells, $C$ is the cell capacitance and $q$ the charge of the carriers. The increase in the breakdown voltage reduces the gain of the individual cells and this further reduces gain with increasing temperature. The results shown in Figs. 7 and 8 indicated that for the scintillator employed here, and for the 400 and 1600 microcells detectors, there is a wide enough range of temperatures within which the devices can be operated in order to detect the $511 \mathrm{keV}$ peak without significant change in energy resolution and just a modest $(<20 \%)$ variation in gain.

In order to summarize the results obtained with the single SiPMs, we calculated the dependence of the gains for all the studies in both the temperature and voltage:

Gain variation $=\frac{\Delta \text { Gain } / \Delta \text { Voltage }}{\overline{\text { Gain }}} \times 100\left(\% \mathrm{~V}^{-1}\right)$

and a similar equation for the variation with temperature. Table 3 shows the gain variation as defined in Eq. (3) with the voltage or the temperature.

\section{Results for the SiPM array}

Fig. 9 shows the four signals produced by an event acquired with the $2 \times 2$ array. The preamplifier is somewhat slower in this case introducing some shaping so that pulses decay time is now about $200 \mathrm{~ns}$. The amplitude at the preamplifier output is between 50 and $450 \mathrm{mV}$, depending on the energy of the photon interacting with the scintillator. These pulses are integrated and the interaction point is estimated using an "Anger logic" to compute the centroid [17].

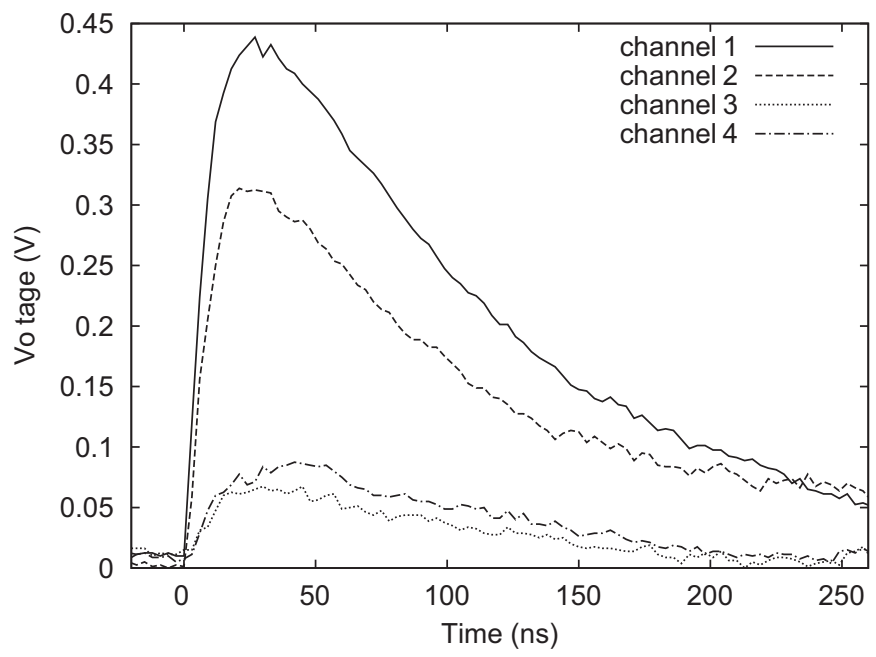

Fig. 9. Pulses for the four channels corresponding to one event detected with the SiPM array.

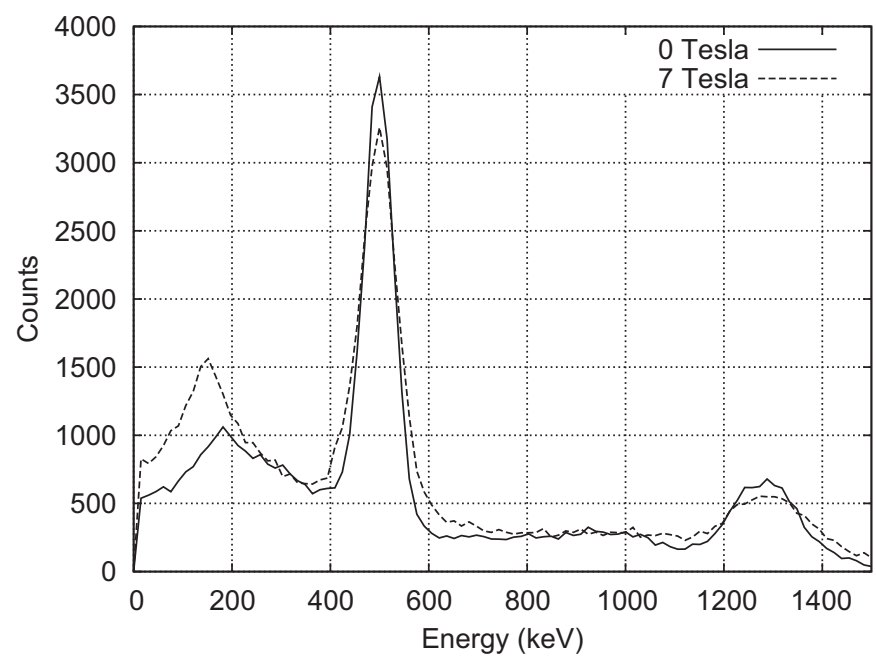

Fig. 10. Energy spectra obtained for the SiPM array coupled to the LYSO crystal using a ${ }^{22} \mathrm{Na}$ radioactive source, after linearizing them according to Eq. (1).

\subsection{Static magnetic field}

Fig. 10 shows the energy spectra obtained with the SiPM array coupled to the single block LYSO crystal using a ${ }^{22} \mathrm{Na}$ radioactive source, both with and without magnetic field. As before, these spectra have been linearized [10] according to Eq. (1). These two spectra show slightly different energy resolution probably due to the variation in the optical coupling between crystal and SiPM. We can observe perfectly both the 511 and $1275 \mathrm{keV}$ peaks of ${ }^{22} \mathrm{Na}$, thanks to the higher number of cells available in this larger detector.

Fig. 11 shows the variation of the relative gain for the $2 \times 2$ array SiPM, coupled to the single block LYSO crystal, as a function of overvoltage and temperature, with and without magnetic field. Measurements with different bias voltages were obtained at both 0 and $7 \mathrm{~T}$ showing, as for the previously discussed results for the individual SiPM detectors, no significant effect of the magnetic field.

The remaining measurements were performed with the SiPM array coupled to the $4 \times 4$ crystal matrix. The flood field histogram shown in Fig. 12 was obtained during a 5 min acquisition with an ${ }^{18} \mathrm{~F}$ source, accumulating a total of $5 \times 10^{5}$ counts in singles mode. 

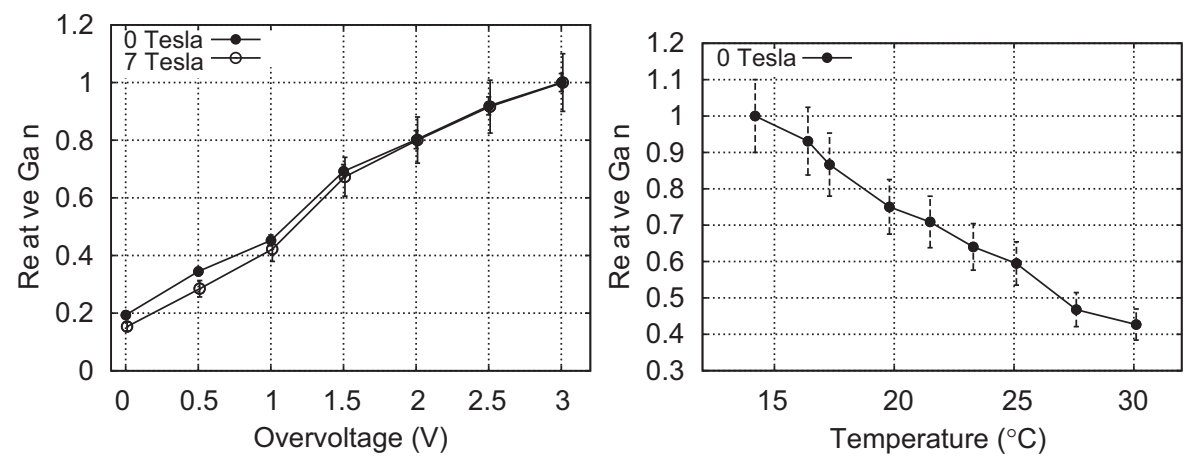

Fig. 11. Relative gain as a function of voltage (left) and temperature (right) for the $2 \times 2$ array SiPM.
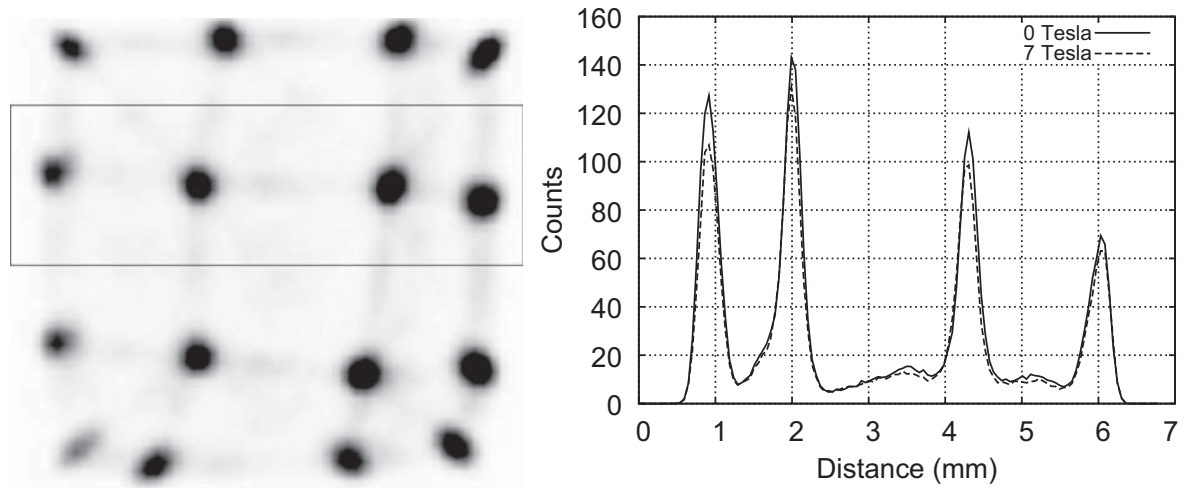

Fig. 12. Flood histogram (left) and horizontal line profile (right) for the $2 \times 2$ array SiPM.

All the crystals of the matrix are clearly resolved. In the same figure, the horizontal profile of counts inside the rectangle of the flood histogram is shown. Solid and dashed lines of the figure present the results obtained at 0 and $7 \mathrm{~T}$, respectively, which yield essentially identical results. During this measurement, a fixed value of the bias voltage of $68.2 \mathrm{~V}$ was applied, while the temperature was kept at $20^{\circ} \mathrm{C}$. A peak to valley ratio of $10: 1$ is achieved. This optimum peak to valley ratio and separation of the crystals is achieved after fine tuning of the bias voltage at the temperature of the current measurement. In a typical case, where deviations of the optimal temperature/bias voltage combination are expected (as in the measurements exhibited in next sections) peak to valley ratios of 7:1 are routinely achieved (Fig. 17).

The energy spectra for ${ }^{18} \mathrm{~F}$, for every crystal element, measured at 0 and $7 \mathrm{~T}$ are shown in Fig. 13.

In Table 4 we show energy resolution at $511 \mathrm{keV}$ for different crystals. It can be seen that crystals in central positions have best resolution, while the ones in the edges and specially the ones in the corners have worse resolution. This is due to incomplete light collection into the detector for the case of edge crystals. As in previous measurements, there is no significant effect of the static magnetic field in these measurements.

\subsection{MR sequence}

Radio frequency (RF) pulses employed in MRI imaging interfere with the readout of the SiPM, introducing noise in the PET signal. This noise may render some SiPM pulses useless, as they will be discarded during Anger like crystal identification or energy windowing procedure, thus introducing a loss of sensitiv ity depending on the particular MRI sequence employed. This

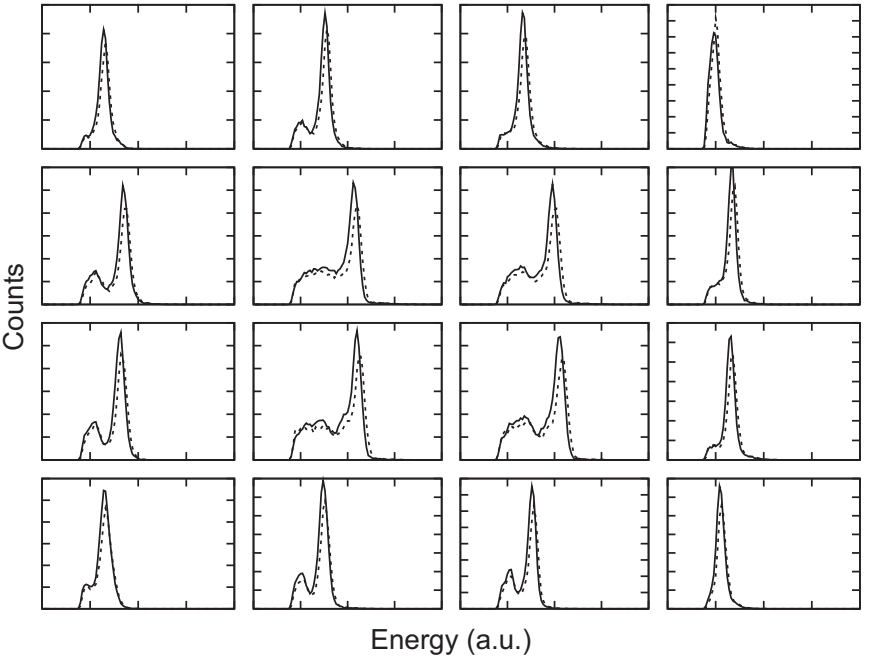

Fig. 13. Energy spectra obtained for each of the sixteen crystal elements, using ${ }^{18} \mathrm{~F}$ when acquiring without (solid lines) and with (dashed lines) magnetic field. These spectra lack the high energy peak of ${ }^{22} \mathrm{Na}$, and it has been acquired with a larger activity source than the other ${ }^{22} \mathrm{Na}$ spectra shown in this work, making the low energy background from intrinsic decay in the scintillator much less noticeable.

Table 4

Energy resolution for crystal element at different positions inside the matrix.

\begin{tabular}{lll}
\hline FWHM (\%) & $0 \mathrm{~T}$ & $7 \mathrm{~T}$ \\
\hline Center & $11 \pm 1$ & $12 \pm 1$ \\
Center edge row & $14 \pm 1$ & $14 \pm 1$ \\
Corner & $22 \pm 2$ & $21 \pm 2$ \\
\hline
\end{tabular}




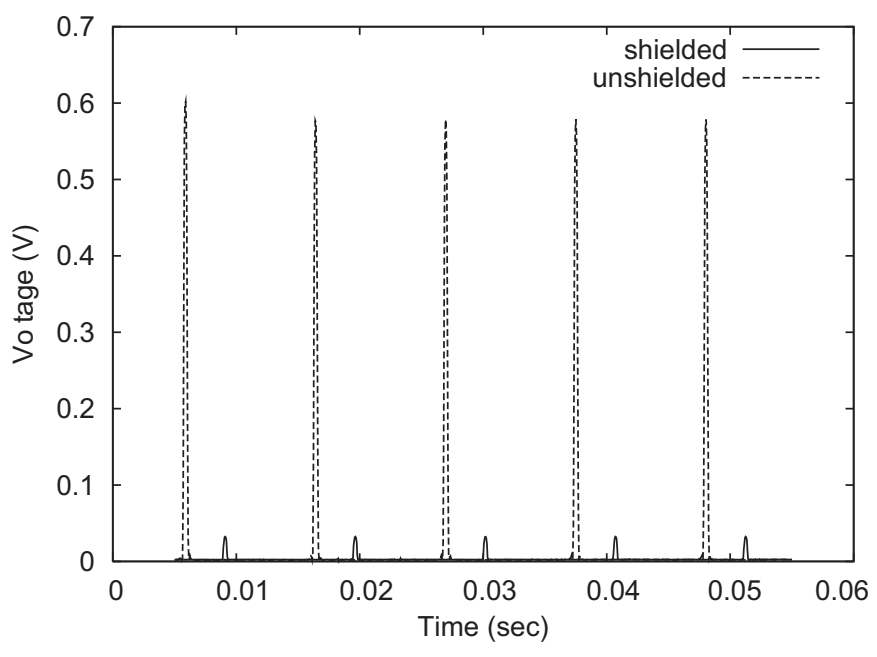

Fig. 14. RF pulses signal induced on the SiPM read-out. Measurements with shielded (solid) and unshielded (dashed) configuration of the readout circuit are shown. Both signals are time shifted in order to disentangle them.

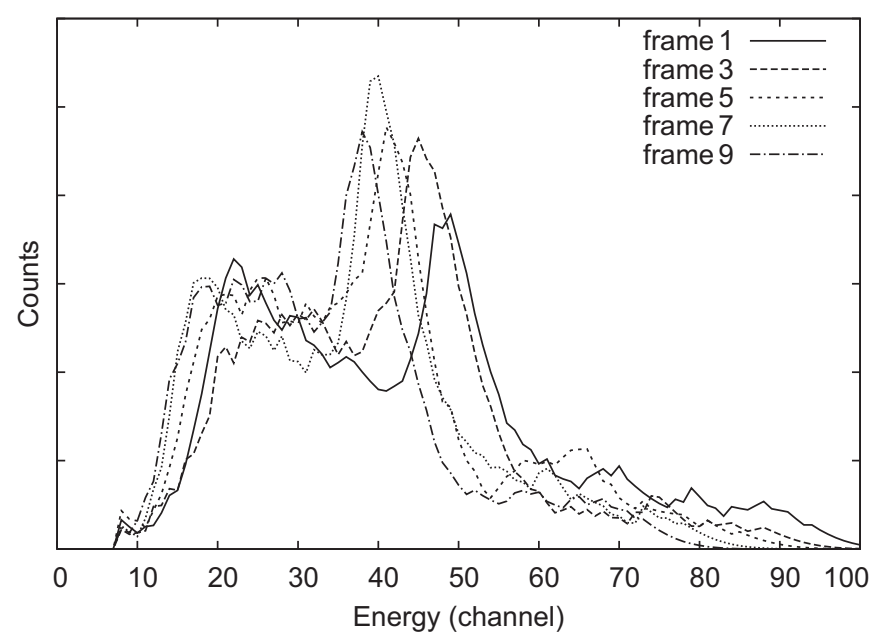

Fig. 15. Energy spectra obtained from a ${ }^{22} \mathrm{Na}$ point source of one crystal element for different frames of the acquisition. Note that whereas acquisition is progressing, the photopeak shifts to the left due to the increase in temperature. The overall acquisition was split in 9 frames of $100 \mathrm{~s}$ of duration, all of them with the same number of counts.

interference from RF pulses is largely reduced by covering the readout circuit of the SiPM with the copper shielding mentioned in the materials and methods section, although this shielding does not completely remove the interference (Fig. 14).

Several $15 \mathrm{~min}$ acquisitions with a $1 \mu \mathrm{Ci}{ }^{22} \mathrm{Na}$ point source were made, recording about 50,000 counts per frame. A high duty MRI sequence, as described before, was applied during acquisition of the gamma signal. When RF was present, we observed a shift in the position of the photopeak. This shift is due to the variation of the gain induced with the change in the temperature, experienced by the electronic circuit when RF is present. A warming up of approximately $10^{\circ} \mathrm{C}$ was observed. At the beginning of the acquisition, the SiPM device is in thermal equilibrium with room temperature (about $20^{\circ} \mathrm{C}$ ) but when the RF sequence begins, the temperature of the device rises and therefore the gain is reduced (Fig. 15). One can see how the energy spectra show clearly the $511 \mathrm{keV}$ peak. Notice that this energy spectrum shows also the contribution from the ${ }^{22} \mathrm{Na}$ high energy photons, and being obtained with a relatively low activity source, it also shows

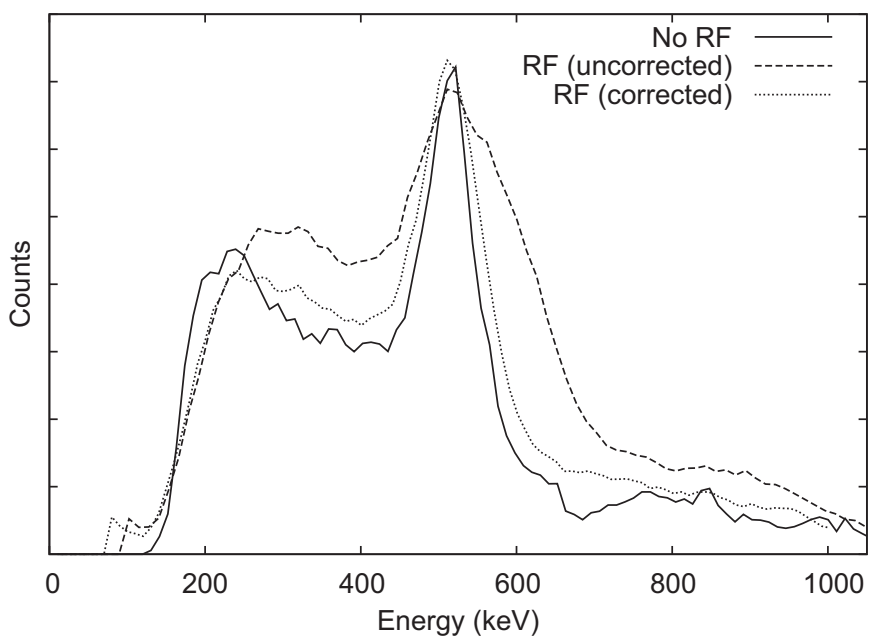

Fig. 16. Energy spectrum of one crystal element acquired without RF (solid) and with RF for the uncorrected (dashed) and gain corrected (dotted) cases.

contribution from the, low energy, intrinsic gamma emission of lutetium.

To remove the changes in gain/temperature from the acquisi tion, and as these changes are smooth over time, we can correct the variation of gain during acquisition of the data by splitting the whole acquisition into shorter frames. The energy spectrum of each frame can be analyzed separately and the annihilation peak can be identified in each of these frames. Once the spectra are properly aligned to yield the same position of the photopeak, we obtain an energy spectrum corrected for the variation of gain (Fig. 16).

In the presence of RF pulses, the signals were acquired with two different bandwidths. In one case, the whole unfiltered bandwidth of the preamplifier (approx. $0.6 \mathrm{GHz}$ ) was employed. In the other case, a low pass filter at $20 \mathrm{MHz}$ was applied. For the unfiltered acquisition, some artifacts can be observed in the flood histogram (Fig. 17) as well as an increase of low energy noise in the energy spectra. Fig. 18 shows the energy spectra obtained with both methods, together with those obtained without RF.

The percentage of counts lost due to energy and position cuts for both filtered and unfiltered acquisition was different. For the unfiltered acquisition, no counts were lost during acquisition compared to the acquisition without RF, but $28 \%$ counts were discarded, compared to the acquisition without $\mathrm{RF}$, after including software cuts in energy window and look up table positioning. In the case of the filtered acquisition, approximately $10 \%$ of counts were lost during the acquisition, compared to the unfiltered case, whereas only $10 \%$ of counts were lost after applying software thresholds in energy compared to the acquisition without RF. Most important is the fact that the filtered acquisition shows no artifacts in the flood field image and a better peak to valley ratio.

\section{Conclusions}

Single SiPMs with an active area of $1 \times 1 \mathrm{~mm}^{2}$ have been tested at 0 and $7 \mathrm{~T}$. We found no significant influence of static magnetic field up to $7 \mathrm{~T}$, obtaining very similar energy resolution (Fig. 5) and identical slopes of gain against voltage (Fig. 6). For these small detectors, the 400 and 1600 microcell SiPMs seem more suitable for PET purposes, exhibiting better and more stable energy resolution (Table 3 ) and gain. 


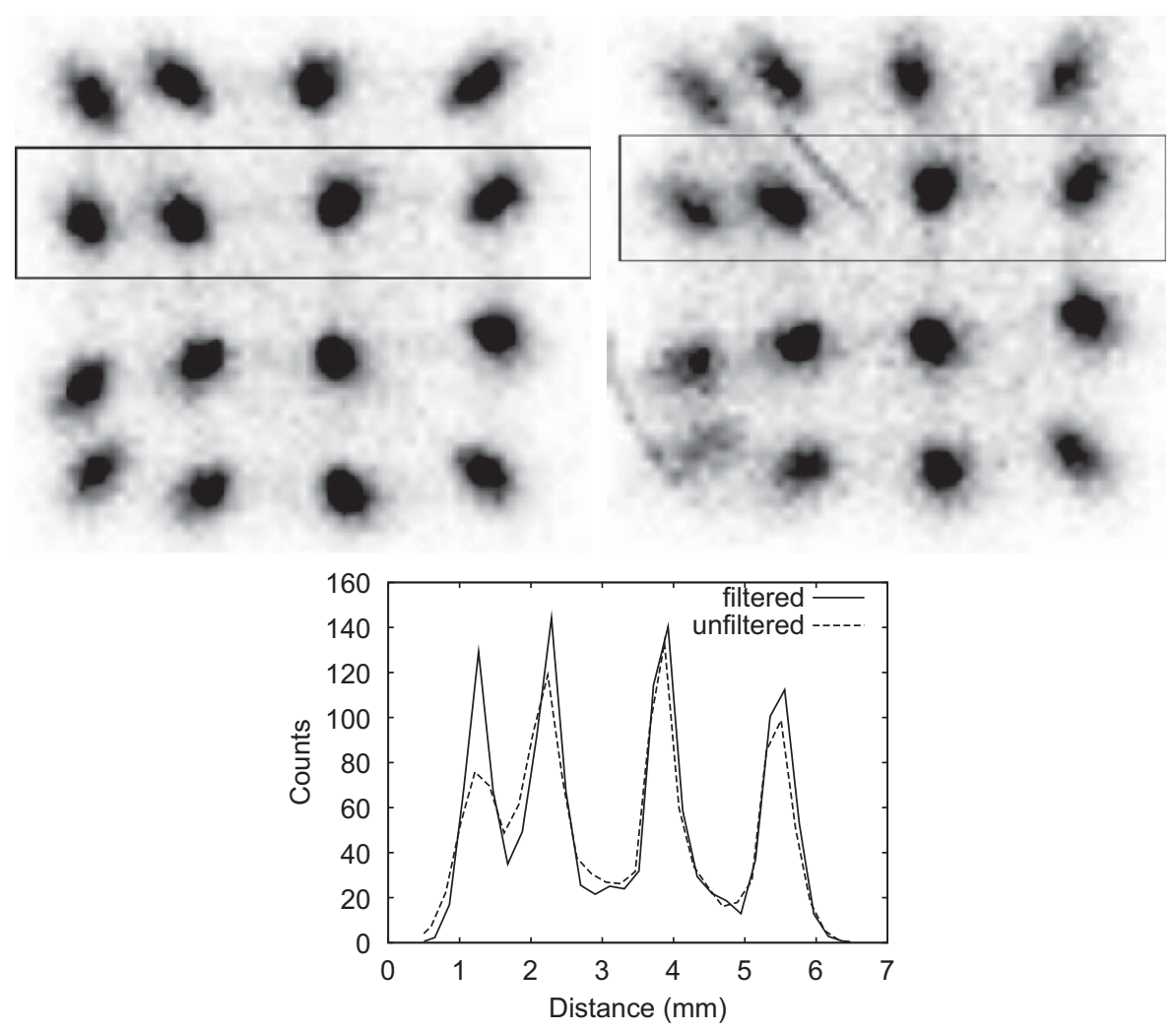

Fig. 17. Flood histograms and line profiles across selected regions when limiting (top) or not (bottom) the bandwidth of the PET signal.
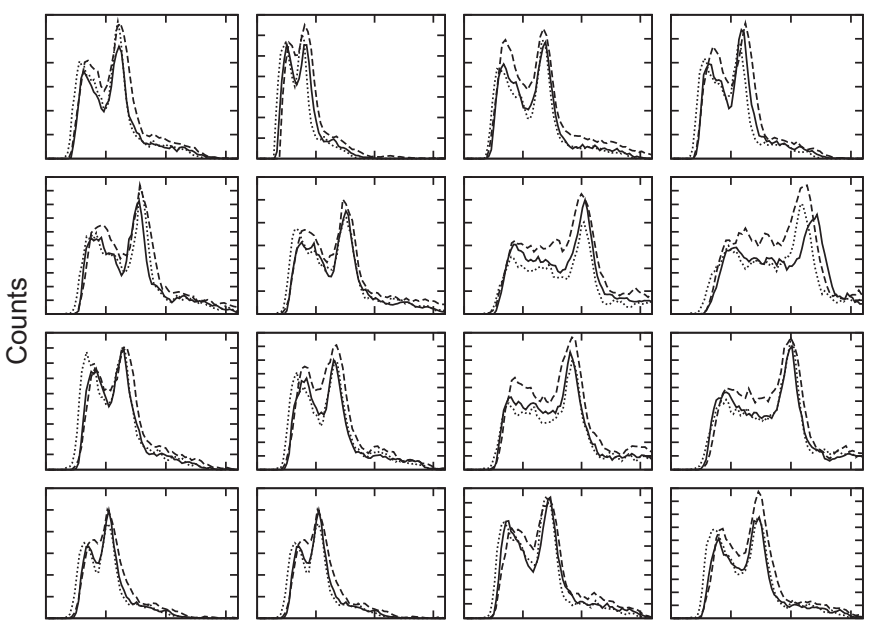

Energy (a.u.)

Fig. 18. Gain corrected energy spectra for each crystal element of the matrix when acquired without RF (solid) and with RF, with raw PET signal (dashed) and with bandwidth limited (dotted) one. In the three cases the spectra show a similar shape with a visible annihilation peak. For most crystals, the bandwidth limited spectra is very similar to the one acquired without RF.

A $2 \times 2$ SiPM array has also been examined, coupled to a $4 \times 4$ LYSO crystal matrix and to a single block larger LYSO crystal. All the $4 \times 4$ crystals of $1.5 \mathrm{~mm}$ pitch size were perfectly resolved by the $2 \times 2$ SiPM array, yielding up to a 10:1 peak to valley ratio in the counts profile at both 0 and $7 \mathrm{~T}$ (Fig. 12). This peak to valley ratio is similar to that obtained with conventional PS PMTs [18]. Fig. 13 shows that the differences of results with and without static magnetic field are negligible. Simultaneous acquisition of SiPM signals to RF pulses from MRI imaging led to a loss of counts of the order of $20 \%$ for the heavy duty MRI sequence employed, due to interference with RF. Either shielding or a simple bandwidth limitation render the signals suitable for PET purposes. Our measurements show the huge potential of SiPMs detectors for its use in PET/MR scanners, with scintillator crystal sizes as small as $1 \mathrm{~mm}$.

\section{Acknowledgment}

This work was supported in part by the CDTEAM project of the CENIT program, and by Ministerio de Ciencia e Innovación under projects FPA2007 62216TEC2008 06715 C02 01, UCM (Grupos UCM; 910059), CPAN (Consolider Ingenio 2010) CSPD 2007 00042 projects, and project SENSORCZT S 0505/MAT/000279, Comunidad de Madrid. Part of the computations of this work were done at the "High Capacity Cluster for Physical Techniques" of UCM, funded in part by the UE under the FEDER program and in part by UCM.

\section{References}

[1] J. Gaa, E.J. Rummeny, M.D. Seemann, Eur. J. Med. Res. 9 (2004) 309.

[2] N.L. Christensen, B.E. Hammer, B.G. Heil, K. Fetterly, Phys. Med. Biol. 4 (1995) 691.

[3] Y. Shao, S.R. Cherry, K. Farahani, K. Meadors, S. Siegel, R.W. Silverman, P.K. Marsden, Phys. Med. Biol. 42 (1997) 1965.

[4] B. Pichler, E. Lorenz, R. Mirzoyan, W. Pimp, F. Roder, M. Schwaiger, S.I. Ziegler, IEEE NSS/MIC Conference Record 2 (1998) 1237.

[5] D. Renker, Nucl. Instr. and Meth. A 567 (2006) 48

[6] A.N. Otte, J. Barral, B. Dolgoshein, J. Hose, S. Klemin, E. Lorenz, R. Mirzoyan, E. Popova, M. Teshima, Nucl. Instr. and Meth. A 545 (2005) 705.

[7] G. Llosá, N. Belcari, M.G. Bisogni, G. Collazuol, A. Del Guerra, S. Marcatili, S. Moehrs, C. Piemonte IEEE NSS/MIC Conference Record 5 (2007) 3220.

[8] D.R. Schaart, H.T. Dam, S. Seifert, R. Vinke, P. Dendooven, H. Löhner, F.J. Beekman, Phys. Med. Biol. 51 (2009) 3501. 
[9] S. Espana, G. Tapias, L.M. Fraile, J.L. Herraiz, E. Vicente, J.M. Udías, M. Desco, J.J. Vaquero, IEEE NSS/MIC Conference Record (2008) 3591.

[10] Hamamatsu, Solid State Division. MPPC Multi-Pixel Photon Counter, January 2008

[11] 〈http://www.analog.com/static/imported-files/data_sheets/AD8002.pdf 〉.

[12] J. Seidel, J.J. Vaquero, F. Barbosa, I.J. Lee, C. Cuevas, M.V. Green, IEEE Trans. Nucl. Sci. NS-47 (2000) 1640.

[13] J. Seidel, J.J. Vaquero, S. Siegel, W.R. Gandler, M.V. Green, IEEE Trans. Nucl. Sci. NS-46 (1999) 485.
[14] D.J. Herbert, D. D’Ascenzo, N. Belcari, A. del Guerra, F. Morsani, V. Saveliev Nucl. Instr, and Meth. A 567 (2006) 356.

[15] A. Goetzberger, B. McDonald, R.H. Haitz, R.M. Scarlett, J. Appl. Phys. 34 (1963) 1591.

[16] S. Gomi, et al., Nucl. Instr. and Meth. A 581 (2007) 427.

[17] H.O. Anger, Kaku Igaku 62 (1969) 125.

[18] J.J. Vaquero, J. Seidel, S. Siegel, W.R. Gandler, M.V. Green, IEEE Trans. Med. Imag. 17 (1998) 967. 